\title{
Simultaneous calculation of momentum and position of an electron present inside p-orbital of the atom
}

\author{
Ravin Kumar ${ }^{[0000-0002-3416-2679]}$ \\ Department of Computer Science, Meerut Institute of Engineering and Technology, meerut- \\ 250005, Uttar Pradesh, India \\ ravin.kumar.cs.2013@miet.ac.in
}

\begin{abstract}
Heisenberg uncertainty principle suggests that momentum and position of an electron can not be correctly detected simultaneously in an atom. This paper proposes a theory which can be found useful in the simultaneous calculation of the momentum and position of an electron present in the atom.
\end{abstract}

Keywords: Heisenberg Uncertainty Principle, Quantum Superposition, Quantum Entanglement, Atomic Theory, Quantum Mechanics, Chemistry.

\section{Introduction}

Atomic orbitals are used to describe the wave-like behavior of electrons in an atom. These are also used to study the chemical bond formation in a molecule. While, Heisenberg uncertainty principle suggests that position and momentum of an electron can not be calculated simultaneously but this paper proposes a theory which strongly suggests that they both can be calculated simultaneously if the stated conditions are met.

\section{$2 \quad$ Related Works}

In Heisenberg uncertainty principle [1] suggests that momentum and position of an electron in the atom can not be simultaneously calculated. Quantum superposition [2] and quantum entanglement [3-4] helped in better understanding the substance and their interactions at the very smaller but interesting scale. Hadamard gate [5] is used in quantum circuit for performing quantum superposition. Along with Hadamard gate, Controlled Hadamard gate [6], Pauli X [7], Controlled Pauli X gate[8] gate are heavily used to develop quantum circuits for positively or negatively correlated quantum entanglement. Atomic orbitals are used to understand the behavior of electrons, along with other particles present inside the atom at the quantum level.

\section{Atomic orbitals}

Atomic orbital model is used to visualize the subatomic behavior of electrons inside an atom. It helps in calculating the probability of finding any electron of an atom in 
any specific region around its nucleus. The s orbital, p orbital, d orbital and f orbital along with the quantum number ' $n$ ' are used to describe the electronic configuration of an atom. The modern form of Aufbau principle is used to predict the approximate electronic configuration of an atom.

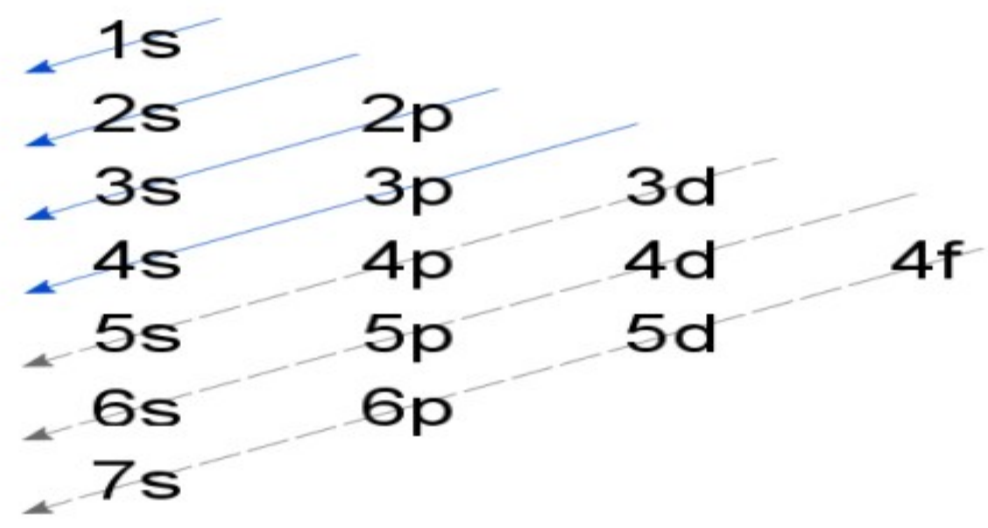

Fig. 2. Hadamard gate applied on $\mid 0>$ input state.

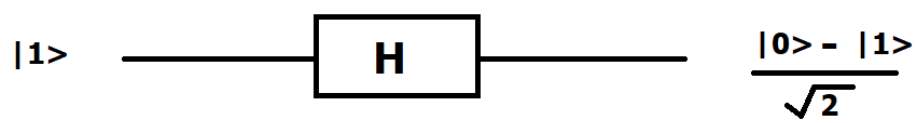

Fig. 3. Hadamard gate applied on $\mid 1>$ input state

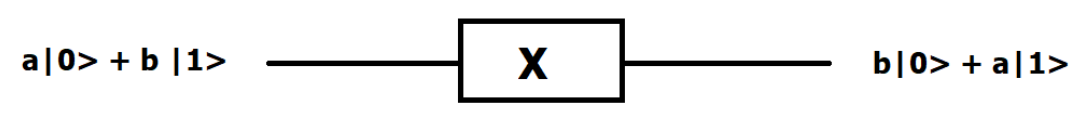

Fig. 4. Pauli X gate interchanging the amplitudes of input state.

Controlled-Pauli X gate consists of a control bit which when equals $\mid 1>$ perform Pauli $\mathrm{X}$ operation on the target bit (Fig. 5), similarly the Controlled-Hadamard gate consists of a control bit which when equals $\mid 1>$ performs Hadamard operation on the target bit (Fig. 6). 


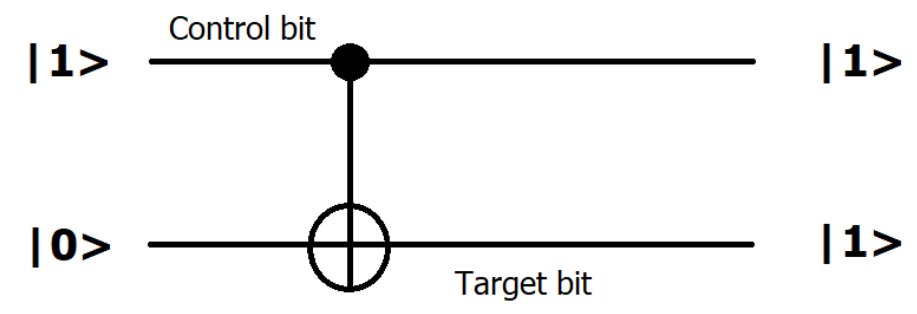

Fig. 5. Controlled-Pauli X gate with input $\mid 1>$ to control bit, and input $\mid 0>$ to target bit.

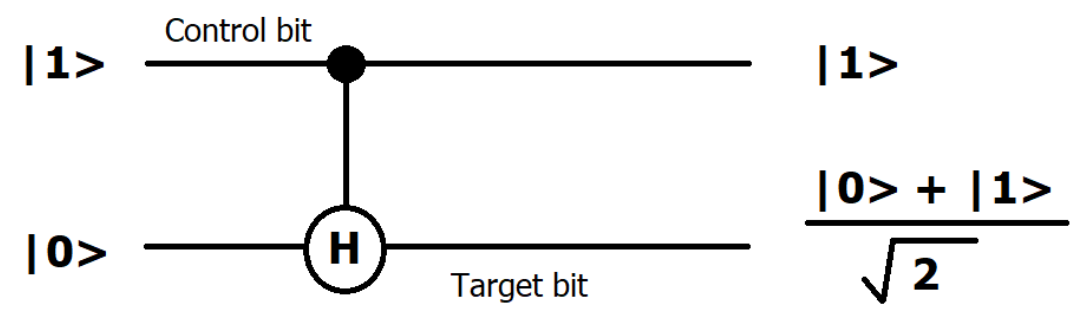

Fig. 6. Controlled-Hadamard gate with input $\mid 1>$ to control bit, and input $\mid 0>$ to target bit.

Hadamard, Pauli X, and their Controlled gates are used to create positively correlated quantum entanglement (Fig. 7) and negatively correlated quantum entanglement (Fig. 8). In positively correlated entanglement, both output bits provide same results when measured, while in a negatively correlated entanglement, output bits always provide opposite states when measured. The interesting thing is that output state is revealed only at the time of measurement. 


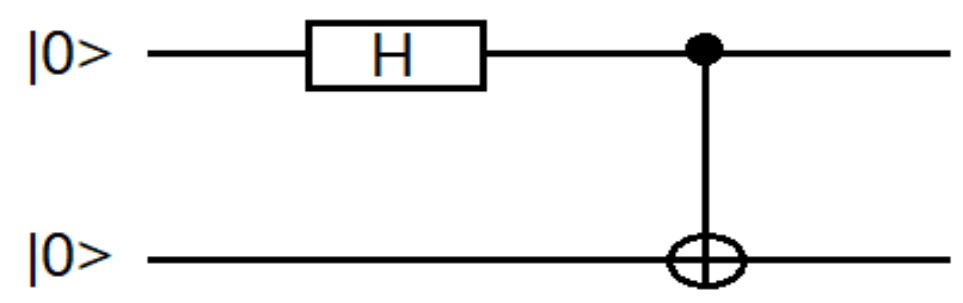

Fig. 7. Positive correlation between two entangled qubits..

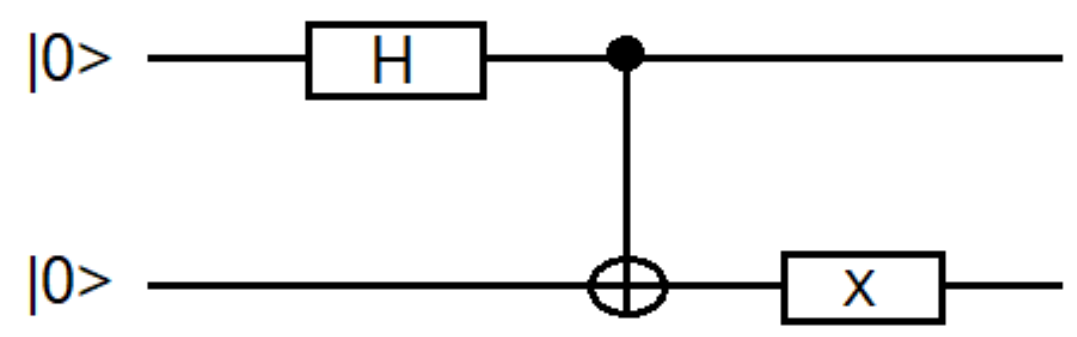

Fig. 8. Negative correlation between two entangled qubits.

\section{Our Proposed Theory}

We propose a theory which when experimentally verified, can be used for the simultaneous calculation of momentum and position of an electron present in the atom. In this theory we suggest that when an atomic orbital has a pair of electrons in it, then they both are entangled such that position and momentum of the electron can be calculated by simultaneously calculating the momentum of the first electron, and position of the second electron of the same atomic orbital. Then the position of the first electron can be calculated using the measured position of the second electron (when found inside the lobe).

In an orbital consisting of two electrons of opposite spin, the position of any one of the electrons can be calculated using the observed position of the other electron. The position of one electron is equal to the refraction of the position (measured from the center of the nucleus) of the other electron (electron is found inside a lobe) across the plane of symmetry. Here plane of symmetry is defined such that it separates the atomic orbital into equally shaped distinguishable lobes (with complementary spins) for the $\mathrm{p}$ atomic orbitals. 


\subsection{Calculating the momentum of the first electron of the atomic orbital}

Simultaneously measure the momentum of the first electron present inside the energy orbital.

\subsection{Calculating the position of the second electron of the same atomic orbital}

Simultaneously measure the position of the second electron present in the same atomic orbital. The measured position of the second electron can be used to calculate the position of the first electron.

Our theory is based on the assumption that measurement equipment is able to simultaneously measure the momentum for one electron, and position for the other. Otherwise after the first measurement, both the electrons will again go back in superposition and when the second measurement will be made then both the electrons will again form from the electron cloud and the experiment will probably give wrong measurement.

\section{Sample Demonstration}

Let us consider an electronic configuration where an individual p-orbital ( $\mathrm{x} \mathrm{X,} \mathrm{p} \mathrm{Y} \mathrm{or}$ $\mathrm{p} Z$ ) contains two electrons. Two simultaneous measurements are performed, one for measuring the position $\mathrm{P}(\mathrm{x} 1, \mathrm{y} 1, \mathrm{z} 1)$ of an electron inside the upper lobe in $\mathrm{P} \mathrm{x}$-orbital, and other for measuring the momentum of the electron inside the lower lobe. We can calculate the position $\mathrm{Q}$ of the second electron using the measured position $\mathrm{P}(\mathrm{x} 1, \mathrm{y} 1, \mathrm{z} 1)$ of the first electron. Calculate the refraction of point $\mathrm{P}(\mathrm{x} 1, \mathrm{y} 1, \mathrm{z} 1)$ about the $y-z$ plane (plane of symmetry). It provides position of the electron in the lower lobe as the point $\mathrm{Q}(-\mathrm{x} 1,-\mathrm{y} 1,-\mathrm{z} 1)$.

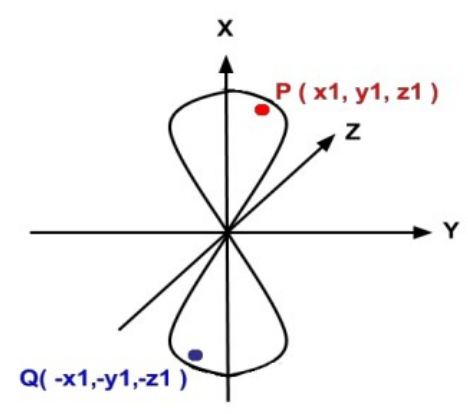

Fig. 9. Predicting position $Q$ of an electron using the observed position $P$ of the first electron (inside the p-orbital containing two electrons in opposite spins). 


\section{Conclusion}

Our proposed theory after experimental verification, will create a better understanding of the atom and its sub atomic particles. It will help in new discoveries in the study of atom specially for the deeper understanding of chemistry at the quantum level.

\section{References}

1. Heisenberg, W. "Uber den anschaulichen Inhalt der quantentheoretischen Kinematik and Mechanik." Zeits. f. Physik 43 (1927):172-198. English translation in WZ,148 pp. 62-84.

2. de Ronde C. Quantum superpositions and the representation of physical reality beyondmeasurement outcomes and mathematical structures. Foundations of Science. $2016 \mathrm{Mar}$ 19:1-28.

3. Witten E. Notes on some entanglement properties of quantum field theory. arXiv preprint arXiv:1803.04993. 2018 Mar 13.

4. Hadjiivanov L, Todorov I. Quantum entanglement. arXiv preprint arXiv:1506.04262. 2015 Jun 13.

5. Tipsmark A, Dong R, Laghaout A, Marek P, Ježek M, Andersen UL. Experimental demonstration of a Hadamard gate for coherent state qubits. Physical Review A. 2011 Nov 8;84(5):050301.

6. Vishnu PK, Joy D, Behera BK, Panigrahi PK. Experimental demonstration of non-local controlled-unitary quantum gates using a five-qubit quantum computer. Quantum Information Processing. 2018 Oct 1;17(10):274.

7. Williams CP. Quantum Gates. In Explorations in Quantum Computing 2011 (pp. 51-122). Springer, London.

8. Saeedi M, Zamani MS, Sedighi M. Algebraic characterization of CNOT-based quantum circuits with its applications on logic synthesis. arXiv preprint arXiv:0712.2963. 2007 Dec 18. 